\title{
Interacting Ghost Dark Energy in Brans-Dicke Theory
}

\author{
Esmaeil Ebrahimi ${ }^{1,2} *$ and Ahmad Sheykhi ${ }^{1,2,3} \dagger$ \\ 1 Department of Physics, Shahid Bahonar University, PO Box 76175, Kerman, Iran \\ 2 Research Institute for Astronomy and Astrophysics of Maragha (RIAAM), Maragha, Iran \\ 3 Physics Department and Biruni Observatory, Shiraz University, Shiraz 71454, Iran
}

\begin{abstract}
We investigate the QCD ghost model of dark energy in the framework of Brans-Dicke cosmology. First, we study the non-interacting ghost dark energy in a flat Brans-Dicke theory. In this case we obtain the EoS and the deceleration parameters and a differential equation governing the evolution of ghost energy density. Interestingly enough, we find that the EoS parameter of the non-interacting ghost dark energy can cross the phantom line $\left(w_{D}=-1\right)$ provided the parameters of the model are chosen suitably. Then, we generalize the study to the interacting ghost dark energy in both flat and non-flat Brans-Dicke framework and find out that the transition of $w_{D}$ to phantom regime can be more easily achieved for than when resort to the Einstein field equations is made.
\end{abstract}

\section{INTRODUCTION}

Many observational evidences such as data from type Ia supernovae [1], cosmic microwave background (CMB) [2, 3] and SDSS [4, 5] have convinced people to accept that our universe is currently experiencing a phase of accelerated expansion. Based on the Einstein's theory of gravity we should introduce a new type of energy with a negative pressure to provide such an epoch of evolution. This unknown energy component which push the universe to accelerate usually called dark energy (DE) in the literature. Of course, the simplest candidate for DE is the cosmological constant originally introduced by Einstein to explain the static behavior of the universe at that time. However, cosmological constant suffers coincidence and fine tuning problems. Also a dynamic approach to dark energy has been proposed avoiding such problems and in favor of experimental evidences seeking a time varying equation of state (EoS) parameter $w_{D}=p_{D} / \rho_{D}$. Examples of such models are quintessence [6, 7], K-essence [8 10], phantom [11], quintom [35], tachyon [13], holographic [14] and agegraphic DE [15].

In recent years there has been a new attention to the so called "scalar-tensor gravity". Scalar-tensor models of gravity are those introduce a scalar field modifying the Einstein's theory of gravity. These models are mainly seen to retrieve at the low energy limit of string theory. However, the history of these models back to many years ago. The scalar tensor theories have started with the work by P. Jordan in 1950 [16]. A prototype of such models was proposed by Brans and Dicke in 1961 [17]. Their aim for presenting this model was to modify Einstein's theory in a way admitting the so called "Mach's principle". To this end they proposed a new scalar degree of freedom to incorporate the Mach's principle into general relativity [17]. The Brans-Dicke (BD) theory can pass the experimental tests from the solar system [18] and provide an explanation for the acceleration of the universe expansion [19].

Most DE models introduce new degrees of freedom in addition to those exist in the standard model of cosmology. Introducing such degrees of freedom need investigation about their nature and new consequences in the universe. Hence, it seems so impressive and economic if one can explain the DE puzzle using already presented fluids and fields in the literature. Ghost dark energy (GDE) is an example of these models which uses the so called Veneziano ghost field to explain the recent acceleration of the universe 20, 21]. Originally the so called "Veneziano ghost field" was presented as a solution to $U(1)$ problem in effective low energy QCD 22 25]. Taking into account the ghost field leads some consequences in the vacuum energy density in a dynamic spacetime or a spacetime with nontrivial topology, while such a field seems to be un-physical in the Minkowski spacetime and has no contribution to the vacuum energy density. In curved spacetime the ghost field gives rise to a small vacuum energy density proportional to $\Lambda_{Q C D}^{3} H$, where $H$ is the Hubble parameter and $\Lambda_{Q C D}^{3}$ is QCD mass scale [21]. It is shown that such a vacuum energy density is capable to drive a phase of acceleration and can be considered as a dynamical cosmological constant [26, 27]. It is important to note that Freidmann equation for GDE model in Einstein gravity is similar to the self-accelerating branch of the Dvali-Gabadadze-Porrati (DGP) braneworld [28, 29], that is both of them have the form $H^{2} \propto \alpha H+\rho_{M}$, [21, 30]. However we would like to emphasize here that in DGP braneworld the term proportinal to $H$ appears due to the inclusion of large extra dimension in the gravity theory and hence modification of the Einstein field equations which yields an extra degree of freedom in the theory, while GDE model is totally embedded in standard model and general relativity, one needs not to introduce any new parameter, new degree of freedom or to modify gravity. As

\footnotetext{
* eebrahimi@uk.ac.ir

$\dagger$ sheykhi@uk.ac.ir
} 
a result the origin of the term $\alpha H$ in GDE model completely differs from previous models such as self-accelerating DGP braneworld scenario [30].

Although, it is a general belief that the current curvature of the universe is negligible and mostly the universe is considered with a flat geometry, recent observations support the possibility of a non-flat universe and detect a small deviation from $k=0$ [31]. For example evidences from CMB and also supernova measurements of the cubic correction to the luminosity distance favor a positively curved universe [32, 33]. In addition, some exact analysis of the WMAP data reveals the possibility of a closed universe [34].

Various aspects of GDE have recently investigated. A generalization of GDE model in a non-flat universe was discussed in [35]. Tachyon and quintessence reconstruction of GDE model were studied in [36, 37]. Since GDE model belongs to a dynamical cosmological constant, it is more natural to study it in the framework of BD theory than in Einstein gravity. In this paper, we study a cosmological model of late acceleration based on the GDE model in the framework of non-flat BD cosmology.

This paper is organized as follows. In the next section, we present the GDE model in the flat BD theory. Interacting GDE model in a flat BD theory is discussed in section III. In section IV, we generalize the study to the universe with spatial curvature. We summarize our results in section $\mathrm{V}$

\section{GHOST DARK ENERGY IN BD THEORY}

The action of BD theory, in the canonical form, can be written [38]

$$
S=\int d^{4} x \sqrt{g}\left(-\frac{1}{8 \omega} \phi^{2} R+\frac{1}{2} g^{\mu \nu} \partial_{\mu} \phi \partial_{\nu} \phi+L_{M}\right),
$$

where $R$ is the scalar curvature and $\phi$ is the BD scalar field. The non-minimal coupling term $\phi^{2} R$ replaces with the Einstein-Hilbert term $R / G$ in such a way that $G_{\text {eff }}^{-1}=2 \pi \phi^{2} / \omega$, where $G_{\text {eff }}$ is the effective gravitational constant as long as the dynamical scalar field $\phi$ varies slowly. In the Jordan frame, the matter minimally couples to the metric and there is no interaction between the scalar field $\phi$ and the matter fields. The equations of motion for the metric $g_{\mu \nu}$ and the BD scalar field $\phi$ are

$$
\begin{aligned}
& \phi G_{\mu \nu}=-8 \pi T_{\mu \nu}^{M}-\frac{\omega}{\phi}\left(\phi_{, \mu} \phi_{, \nu}-\frac{1}{2} g_{\mu \nu} \phi_{, \lambda} \phi^{, \lambda}\right)-\phi_{; \mu ; \nu}+g_{\mu \nu} \square \phi, \\
& \square \phi=\frac{8 \pi}{2 \omega+3} T_{\lambda}^{M \lambda},
\end{aligned}
$$

where $T_{\mu \nu}^{M}$ is the matter energy-momentum tensor. Our aim in this paper is to consider the GDE in the FriedmannRobertson-Walker (FRW) universe which is described by the line element

$$
d s^{2}=d t^{2}-a^{2}(t)\left(\frac{d r^{2}}{1-k r^{2}}+r^{2} d \Omega^{2}\right),
$$

where $a(t)$ is the scale factor, and $k$ is the curvature parameter with $k=-1,0,1$ corresponding to open, flat, and closed universes, respectively. A closed universe with a small positive curvature $\left(\Omega_{k} \simeq 0.01\right)$ is compatible with observations [39]. Using metric (4), the field equations (2) and (3) reduce to

$$
\begin{aligned}
& \frac{3}{4 \omega} \phi^{2}\left(H^{2}+\frac{k}{a^{2}}\right)-\frac{1}{2} \dot{\phi}^{2}+\frac{3}{2 \omega} H \dot{\phi} \phi=\rho_{M}+\rho_{D}, \\
& \frac{-1}{4 \omega} \phi^{2}\left(2 \frac{\ddot{a}}{a}+H^{2}+\frac{k}{a^{2}}\right)-\frac{1}{\omega} H \dot{\phi} \phi-\frac{1}{2 \omega} \ddot{\phi} \phi-\frac{1}{2}\left(1+\frac{1}{\omega}\right) \dot{\phi}^{2}=p_{D}, \\
& \ddot{\phi}+3 H \dot{\phi}-\frac{3}{2 \omega}\left(\frac{\ddot{a}}{a}+H^{2}+\frac{k}{a^{2}}\right) \phi=0,
\end{aligned}
$$

where $H=\dot{a} / a$ is the Hubble parameter, $\rho_{D}$ and $p_{D}$ are, respectively, the energy density and pressure of DE, and $\rho_{M}$ is the pressureless dark matter (DM) density.

Consider the FRW universe filled with DE and pressureless matter which evolves according to their conservation laws

$$
\begin{aligned}
& \dot{\rho}_{D}+3 H \rho_{D}\left(1+w_{D}\right)=0, \\
& \dot{\rho}_{M}+3 H \rho_{M}=0,
\end{aligned}
$$


where $w_{D}$ is the EoS parameter of DE. In this section we want to consider the GDE in a spatially flat spacetime in the BD framework. The ghost energy density is proportional to the Hubble parameter [21]

$$
\rho_{D}=\alpha H
$$

Here $\alpha$ is a constant of order $\Lambda_{\mathrm{QCD}}^{3}$ where $\Lambda_{\mathrm{QCD}} \sim 100 \mathrm{MeV}$ is QCD mass scale. With $H \sim 10^{-33} \mathrm{eV}, \Lambda_{\mathrm{QCD}}^{3} H$ gives the right order of magnitude $\sim\left(3 \times 10^{-3} \mathrm{eV}\right)^{4}$ for the observed DE density [21].

To determine the evolution of the universe filled by the pressureless matter and GDE, through the equations (5-7), we still have another degree of freedom in analyzing the set of equations. Based on the previous experiences in the $\mathrm{BD}$ theory it is known that usually the BD scalar field $\phi$ has a power law relation as

$$
\phi=\phi_{0} a(t)^{\varepsilon} \text {. }
$$

A case of particular interest is that when $\varepsilon$ is small whereas $\omega$ is high so that the product $\varepsilon \omega$ results of order unity [40]. This is interesting because local astronomical experiments set a very high lower bound on $\omega$ [4]; in particular, the Cassini experiment implies that $\omega>10^{4}$ [18, 42]. Taking the derivative with respect to time of relation (11) we obtain

$$
\frac{\dot{\phi}}{\phi}=\varepsilon \frac{\dot{a}}{a}=\varepsilon H
$$

Using Eqs. (11) and (12), the first Friedmann equation (5) becomes

$$
H^{2}\left(1-\frac{2 \omega}{3} \varepsilon^{2}+2 \varepsilon\right)+\frac{k}{a^{2}}=\frac{4 \omega}{3 \phi^{2}}\left(\rho_{D}+\rho_{M}\right) .
$$

We introduce the fractional energy densities corresponding to each energy component as usual

$$
\begin{aligned}
\Omega_{M} & =\frac{\rho_{M}}{\rho_{\mathrm{cr}}}=\frac{4 \omega \rho_{M}}{3 \phi^{2} H^{2}}, \\
\Omega_{k} & =\frac{\rho_{k}}{\rho_{\mathrm{cr}}}=\frac{k}{H^{2} a^{2}}, \\
\Omega_{D} & =\frac{\rho_{D}}{\rho_{\mathrm{cr}}}=\frac{4 \omega \rho_{D}}{3 \phi^{2} H^{2}},
\end{aligned}
$$

where we have defined

$$
\rho_{\mathrm{cr}}=\frac{3 \phi^{2} H^{2}}{4 \omega}
$$

Using (10) we can rewrite Eq. (16) as

$$
\Omega_{D}=\frac{4 \omega \alpha}{3 \phi^{2} H}
$$

Based on these definitions, Eq. (13) can be rewritten as

$$
\gamma=\Omega_{D}+\Omega_{M}-\Omega_{k}
$$

where

$$
\gamma=1-\frac{2 \omega}{3} \varepsilon^{2}+2 \varepsilon
$$

Taking the time derivative of relation (10), and using the Friedmann equation (13) as well as the continuity equations (8) and (9), we find

$$
\frac{\dot{\rho}_{D}}{\rho_{D}}=\frac{\dot{H}}{H}=-H\left[\varepsilon+\frac{3}{2}+\frac{3}{2} \frac{\Omega_{D} w_{D}}{\gamma}\right] .
$$

Substituting this relation in continuity equation (8) we obtain the EoS parameter of GDE, namely

$$
w_{D}=\frac{\gamma}{2 \gamma-\Omega_{D}}\left[\frac{2 \varepsilon}{3}-1\right]
$$



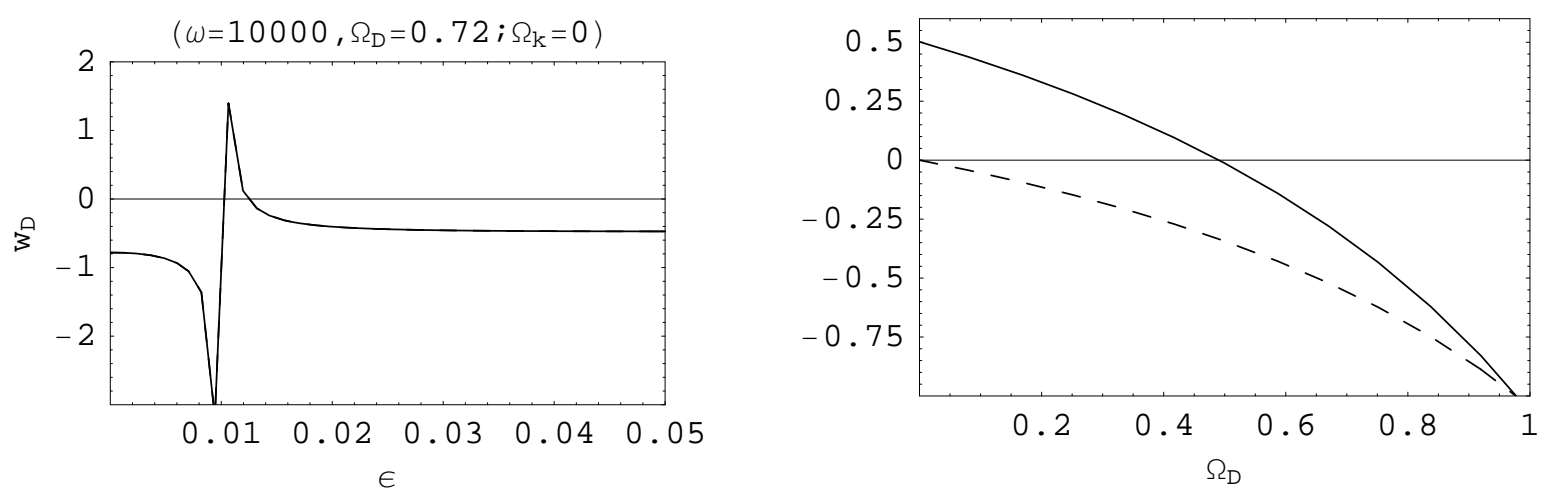

FIG. 1: The first figure indicates $w_{D}$ against $\varepsilon$ in the flat non-interacting GDE. In the second figure the deceleration parameter (solid) and $w_{\text {eff }}$ (dashed) are plotted versus $\Omega_{D}$ for $\varepsilon=0.002$.

It is worthy to note that in the limiting case $\varepsilon=0(\omega \rightarrow \infty)$ we have $\gamma=1$ and hence the BD scalar field becomes trivial; as a result Eq. (22) reduces to its respective expression in flat standard cosmology [27]

$$
w_{D}=-\frac{1}{2-\Omega_{D}} .
$$

The solar-system experiments give the result for the value of $\omega$ is $\omega>40000$ [18]. However, when probing the larger scales, the limit obtained will be weaker than this result. In Ref. [42], the authors found that $\omega$ is smaller than 40000 on the cosmological scales. Also, Wu and Chen [43] obtained the observational constraints on BD model in a flat universe with cosmological constant and cold DM using the latest WMAP and SDSS data. They found that within $2 \sigma$ range, the value of $\omega$ satisfies $\omega<-120.0$ or $\omega>97.8$ [43]. They also obtained the constraint on the rate of change of $G$ at present

$$
-1.75 \times 10^{-12} y r^{-1}<\frac{\dot{G}}{G}<1.05 \times 10^{-12} y r^{-1}
$$

at $2 \sigma$ confidence level. So in our case with assumption (11) we get

$$
\frac{\dot{G}}{G}=\frac{\dot{\phi}}{\phi}=\varepsilon H<1.05 \times 10^{-12} y r^{-1}
$$

This relation can be used to put an upper bound on $\varepsilon$. Assuming the present value of the Hubble parameter to be $H_{0} \simeq 0.7$ we obtain

$$
\varepsilon<0.01
$$

The GDE model in BD framework has an interesting feature compared to the GDE model in Einstein's gravity. It was shown that in standard cosmology based on Einstein's theory, the EoS parameter of the noninteracting GDE cannot cross the phantom line $w_{D}=-1$ and at the late time where $\Omega_{D} \rightarrow 1$ approaches -1 [27]. However, in the $\mathrm{BD}$ framework, requiring condition $w_{D}<-1$ leads to $\gamma\left(\frac{2 \varepsilon}{3}+1\right)<\Omega_{D}$. Choosing $\Omega_{D}=0.72$ for the present time, this inequality valid provided we take $\varepsilon=0.002$ which is consistent with observations. This indicates that one can generate a phantom-like EoS for the noninteracting GDE in the BD framework.

In addition to the EoS parameter of the GDE we can also study the effective EoS parameter, $w_{\text {eff }}$, which is defined as

$$
w_{\mathrm{eff}}=\frac{P_{t}}{\rho_{t}}=\frac{P_{D}}{\rho_{D}+\rho_{M}},
$$

where $\rho_{t}$ and $P_{t}$ are, respectively, the total energy density and total pressure of the universe. As usual we assumed the dark matter is in the form pressureless fluid $\left(P_{M}=0\right)$. Using relation (19) for the flat case one can find

$$
w_{\mathrm{eff}}=\frac{\Omega_{D}}{\gamma} w_{D}=\frac{\Omega_{D}}{2 \gamma-\Omega_{D}}\left[\frac{2 \varepsilon}{3}-1\right]
$$


It is also interesting to study the behavior of the deceleration parameter defined as

$$
q=-\frac{\ddot{a}}{a H^{2}}=-1-\frac{\dot{H}}{H^{2}} .
$$

Inserting from (21) into (29) yields

$$
q=\frac{1}{2}+\varepsilon+\frac{3}{2} \frac{\Omega_{D} w_{D}}{\gamma}
$$

Substituting Eq. (22) in the above relation one can easily reach

$$
q=\frac{1}{2}+\varepsilon+\frac{3 \Omega_{D}}{2\left(2 \gamma-\Omega_{D}\right)}\left(\frac{2 \varepsilon}{3}-1\right) .
$$

Let us study some special cases of interest for the deceleration parameter $q$. If we take $\Omega_{D}=0.72$ for the present time and choosing $\varepsilon=0.002$ and $\omega=10^{4}$ we obtain $q=-0.36$, which is consistent with the present value of the deceleration parameter obtained in [4]. This choice of parameters lead $w_{D}=-0.79$ and $w_{\text {eff }}=-0.58$. The evolution of the deceleration parameter $q$ and $w_{\text {eff }}$ are plotted in the second part of Fig(1). A close look to this figure reveals that the universe enters acceleration phase when $\Omega_{D}=0.49$. The effective EoS parameter at $\Omega_{D}=0.49$ becomes $w_{\text {eff }}=-0.33$ while $w_{D}=-0.66$. We can also study the behavior of the deceleration parameter in the early stage of the universe where $\Omega_{D} \ll 1$. In this epoch $q=\frac{1}{2}+\varepsilon$ which indicates that the universe was experiencing a phase of deceleration at the early stage of its evolution due to the domination of the DM component.

Once again we can see that in the limiting case $\varepsilon=0(\gamma=1)$ the above relation restores the deceleration parameter of GDE in Einstein's gravity [27].

$$
q=\frac{1}{2}-\frac{3}{2} \frac{\Omega_{D}}{\left(2-\Omega_{D}\right)} .
$$

Finally, we obtain a differential equation governing the evolution of GDE from early deceleration to late time acceleration. To do this we take time derivative of the relation (18) and use Eq. (29). The result is

$$
\dot{\Omega}_{D}=\Omega_{D} H(1+q-2 \varepsilon) .
$$

Inserting $q$ from (31) and using relation $\Omega_{D}^{\prime}=H \frac{d \Omega_{D}}{d \ln a}$, we obtain

$$
\Omega_{D}^{\prime}=\Omega_{D}\left[3\left(\frac{1-\Omega_{D}}{2 \gamma-\Omega_{D}}\right)-2 \varepsilon\right],
$$

where prime denotes the derivation with respect to $x=\ln a$. In the limiting case $\varepsilon=0(\gamma=1)$ one recovers the result obtained in [27].

\section{INTERACTING GHOST DARK ENERGY IN FLAT BD THEORY}

Most of the models study the dark side of the universe consider the evolution of DE and DM separately. This means that $\rho_{D M}$ and $\rho_{D E}$ are separately conserved. However, recently there has been a lot of interest in interacting models of DE, since observations detects a signal of interaction between DE and DM. For instance, observational evidences provided by the galaxy cluster Abell A586 supports the interaction between DE and DM [45]. Beside there is no any reason against the interacting behavior of DE and DM and many authors has explained Lagrangians leaded to an interacting approach [46]. Any conservation in the physics can be explained by a symmetry in the Lagrangian level and there does not exist such a symmetry through the known Lagragians explaining the dark side of the universe. These points convince us to discuss a version of GDE in which there exist an interaction between DE and DM. To this end we can write the conservation equation for different components as

$$
\begin{aligned}
& \dot{\rho}_{D}+3 H \rho_{D}\left(1+w_{D}\right)=-Q, \\
& \dot{\rho}_{M}+3 H \rho_{M}=Q,
\end{aligned}
$$

where $Q$ denotes the interaction term and we take it as $Q=3 b^{2} H\left(\rho_{M}+\rho_{D}\right)$ with $b^{2}$ is a coupling constant. Such a choice for interacting term implies the the DE and DM component do not conserve separately while the total density is still conserved through

$$
\dot{\rho}+3 H(\rho+P)=0,
$$



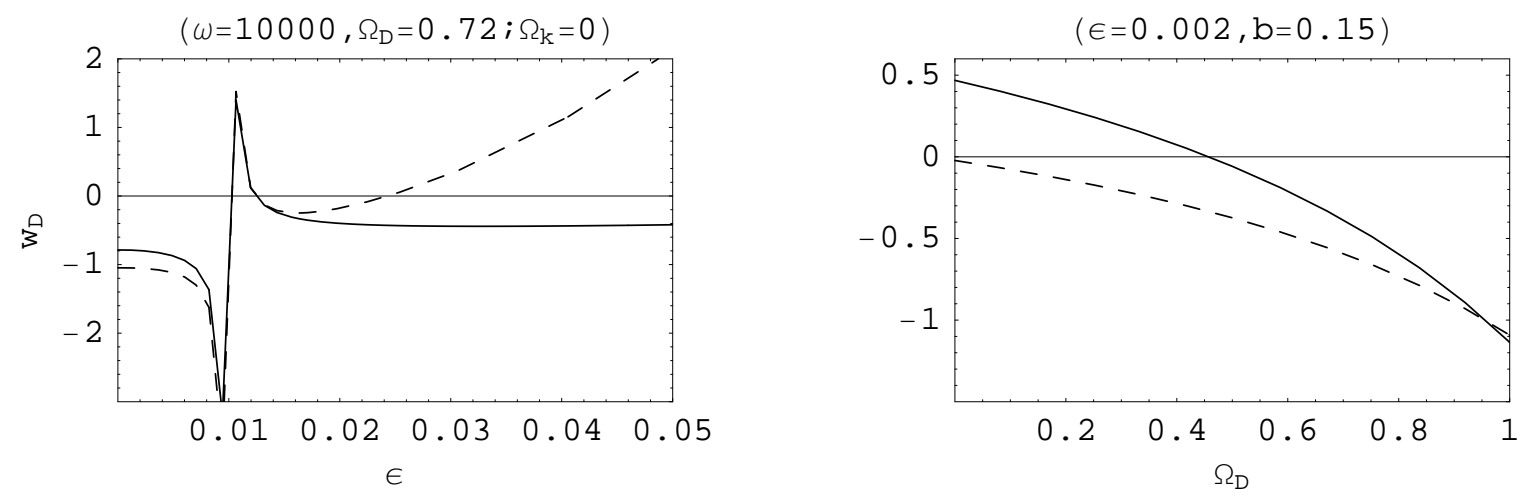

FIG. 2: In the first figure $w_{D}$ is plotted against $\varepsilon$. Solid line corresponds to $b=0.05$ and dashed one for $b=0.2$. In the second figure the deceleration parameter (solid) and the $w_{\text {eff }}$ (dashed) are plotted versus $\Omega_{D}$. These figures are plotted in the flat, interacting GDE case.

where $\rho=\rho_{D}+\rho_{M}$ and $P=P_{D}$. In this section we would like to discuss the interacting GDE in a flat universe. To this goal, once again we take time derivative from (10) to get (21). Next, we obtain the L.H.S. of Eq. (21) from the conservation equation and the R.H.S. from (13) and also taking into account $Q=3 b^{2} H\left(\rho_{M}+\rho_{D}\right)$ and (19) we obtain the EoS parameter

$$
w_{D}=\frac{\gamma}{2 \gamma-\Omega_{D}}\left[\frac{2 \varepsilon}{3}-1-\frac{2 \gamma b^{2}}{\Omega_{D}}\right] .
$$

In the limiting case $\varepsilon=0(\omega \rightarrow \infty)$ or $\gamma=1$, the BD theory reduces to the Einstein gravity and hence Eq. (38) recovers its respective expression in standard cosmology [27]

$$
w_{D}=-\frac{1}{2-\Omega_{D}}\left(1+\frac{2 b^{2}}{\Omega_{D}}\right) .
$$

The effective EoS parameter can be written as

$$
w_{\mathrm{eff}}=\frac{\Omega_{D}}{\gamma} w_{D}=\frac{\Omega_{D}}{2 \gamma-\Omega_{D}}\left[\frac{2 \varepsilon}{3}-1-\frac{2 \gamma b^{2}}{\Omega_{D}}\right] .
$$

In this case, using the method of the previous section, the deceleration parameter is obtained as

$$
q=\frac{1}{2}+\varepsilon+\frac{3 \Omega_{D}}{2\left(2 \gamma-\Omega_{D}\right)}\left[\frac{2 \varepsilon}{3}-\left(1+\frac{2 \gamma b^{2}}{\Omega_{D}}\right)\right] .
$$

In order to obtain an insight about various features of the interacting case, we try a suitable choice of parameters. Taking $\Omega_{D}=0.72, b=0.15$ and $\varepsilon=0.002$, we obtain $q=-0.41$ for the present value of the deceleration parameter which is in good agreement with recent observational results [44]. Also this choice of the parameters result in $w_{D}=-0.84$ and $w_{\text {eff }}=-0.62$ for present time of the universe and one can see the consistency of the latter with current observations. Transition from deceleration to acceleration take places at $\Omega_{D}=0.46$ and at this time $w_{\text {eff }}=-0.33$ while $w_{D}=-0.71$. One may take a look on Fig. 2 for a better insight into the model. The first part of this figure indicates that one can generate a phantom-like behavior provided $\varepsilon<0.01$. Further, we can have a close look at the behavior of $w_{D}$ at the late time where $\Omega_{D} \rightarrow 1$. In this limit and using the same values of $\varepsilon$ and $b$ we find $w_{D}=-1.06$, indicating that the interacting GDE in the BD framework can cross the phantom line in the future. In this limit with a same set of parameters $w_{\text {eff }}=-1.09$ which leads a super acceleration and may result in a big rip as fate of the universe.

The equation of motion of GDE is obtained as

$$
\Omega_{D}^{\prime}=\Omega_{D}\left[\frac{3}{2}\left(1-\frac{\Omega_{D}}{2 \gamma-\Omega_{D}}\left(1+\frac{2 \gamma b^{2}}{\Omega_{D}}\right)\right)-2 \varepsilon\right] .
$$



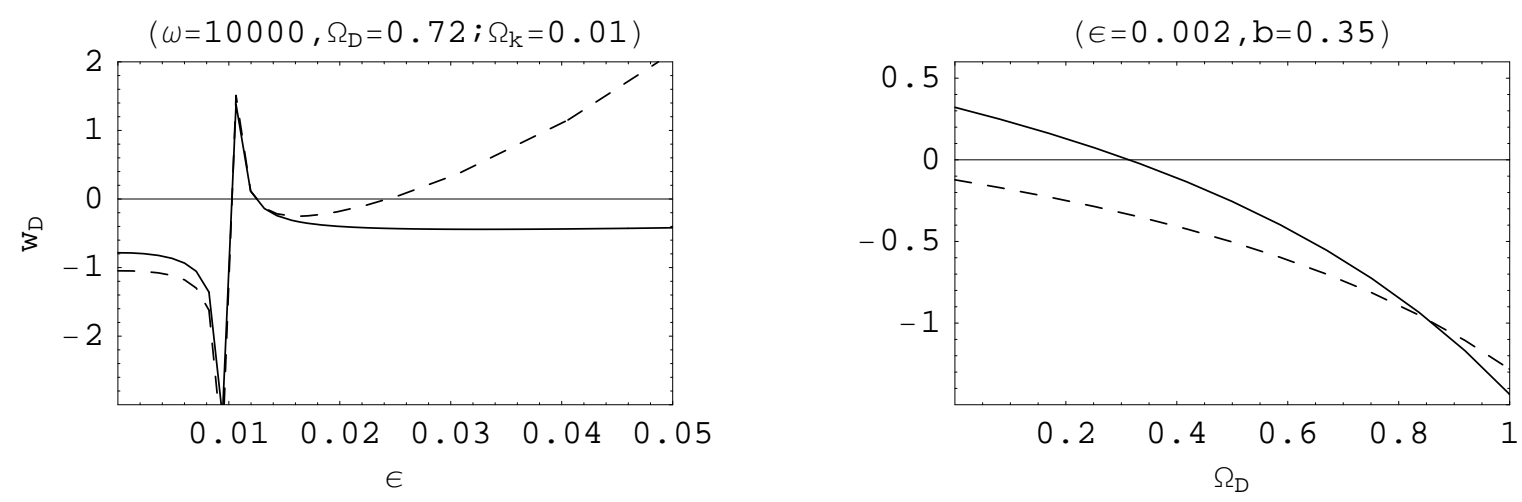

FIG. 3: In this figure $w_{D}$ is plotted against $\varepsilon$. Solid line corresponds to $b=0.05$ and dashed one for $b=0.2$. The second figure shows the evolution of deceleration parameter (solid) and also $w_{\text {eff }}$ (dashed) versus $\Omega_{D}$. These figures are plotted in the presence of the curvature as well as the interaction between DE and DM for GDE in BD framework.

\section{INTERACTING GHOST DARK ENERGY IN NON-FLAT UNIVERSE}

Historically, one of the problems leaded to pioneering theory of inflation was the so-called "flatness problem". flatness problem was explained by assuming a very huge rate of expansion in the first stages of the cosmic evolution namely "inflation". After the inflation it is generally believed that the universe is spatially flat, that is the inflation practically washes out the effect of curvature in the early stages of cosmic evolution. However, in the past decade several observational evidences have been observed in contrast to the flatness assumption of the universe. In the context of inflation it is argued that the flatness is not a necessary consequence of inflation if the number of e-foldings is not very large [47]. Besides, the parameter $\Omega_{k}$ represents the contribution to the total energy density from the spatial curvature and it is constrained as $-0.0175<\Omega_{k}<0.0085$ with $95 \%$ confidence level by current observations [48]. In addition, there are several observational evidences which supports a closed geometry for our universe in 31 34]. Based on these implications it seems interesting to investigate the different DE models while the curvature term is present. So, in this section our main aim is to discuss a non-flat FRW background in the presence of the interacting GDE in BD theory. Taking the curvature into account, the Friedmann equation is rewritten as

$$
\gamma H^{2}+\frac{k}{a^{2}}=\frac{4 \omega}{3 \phi^{2}}\left(\rho_{m}+\rho_{D}\right)
$$

Following the method of the previous section we obtain different parameters in the presence of the curvature term. Taking the time derivative of the Friedmann equation (43) and using (19), we find

$$
\frac{\dot{H}}{H^{2}}=\frac{\Omega_{k}}{\gamma}-\left(1+\frac{\Omega_{k}}{\gamma}\right)\left[\varepsilon+\frac{3}{2}+\frac{3}{2} \frac{\Omega_{D} w_{D}}{\gamma+\Omega_{k}}\right] .
$$

Combining the above equation with Eq. (35), after using Eq. (21), we obtain the EoS parameter as

$$
w_{D}=-\frac{\gamma}{2 \gamma-\Omega_{D}}\left(1-\frac{\Omega_{k}}{3 \gamma}-\frac{2 \varepsilon}{3}\left(1+\frac{\Omega_{k}}{\gamma}\right)+\frac{2 b^{2}}{\Omega_{D}}\left(\gamma+\Omega_{k}\right)\right)
$$

One can easily check that the above equation reduces to the EoS parameter of non-flat interacting GDE in the Einstein's framework provided $\varepsilon=0(\gamma=1)$.

Using the same method as the previous sections, $w_{\text {eff }}$ in a non-flat background reads

$$
w_{\mathrm{eff}}=\frac{\Omega_{D}}{\gamma+\Omega_{k}} w_{D}
$$

This relation can be obtained using Eq.(27) as well as (19) in the non-flat case. Replacing (45) in the above relation, we get

$$
w_{e f f}=-\frac{\gamma \Omega_{D}}{\left(2 \gamma-\Omega_{D}\right)\left(\gamma+\Omega_{k}\right)}\left(1-\frac{\Omega_{k}}{3 \gamma}-\frac{2 \varepsilon}{3}\left(1+\frac{\Omega_{k}}{\gamma}\right)+\frac{2 b^{2}}{\Omega_{D}}\left(\gamma+\Omega_{k}\right)\right)
$$


The deceleration parameter can be calculated by substituting Eqs. (44) and (45) into (29). We find

$$
q=\left(1+\frac{\Omega_{k}}{\gamma}\right)\left[\frac{1}{2}-\frac{2 \gamma \varepsilon}{\Omega_{D}-2 \gamma}\right]+\frac{3 \Omega_{D}}{2\left(2 \gamma-\Omega_{D}\right)}\left[1-\frac{\Omega_{k}}{3 \gamma}+\frac{2 b^{2}}{\Omega_{D}}\left(\gamma+\Omega_{k}\right)\right] .
$$

Also the equation of motion of GDE can be obtained by replacing Eq. (48) in (33). The result is

$$
\frac{d \Omega_{D}}{d \ln a}=\Omega_{D}\left[1+\left(1+\frac{\Omega_{k}}{\gamma}\right)\left[\frac{1}{2}-\frac{2 \gamma \varepsilon}{\Omega_{D}-2 \gamma}\right]+\frac{3 \Omega_{D}}{2\left(2 \gamma-\Omega_{D}\right)}\left[1-\frac{\Omega_{k}}{3 \gamma}+\frac{2 b^{2}}{\Omega_{D}}\left(\gamma+\Omega_{k}\right)\right]-2 \varepsilon\right] .
$$

It is worth to mentioning that all relations we obtained in this section restor their respective expressions in the previous section when we set $\Omega_{k}=0$ as we expect. Furthermore, one can see that the evolution equation of the GDE and the deceleration parameter reduce to the expected formulas in the Einstein's theory [27). A close look to Fig.(3) reveals some interesting features of the model. First of all, we can see that the EoS parameter, $w_{D}$ in all of the figures just can result in an acceleration phase if $\varepsilon<0.01$ which this point is completely consistent with the observational evidences [43]. Additionally one can note that in all cases including flat, non flat, interacting and noninteracting versions of the GDE model, the EoS parameter of GDE can achieve the phantom cross with suitable choices of $\varepsilon$ and the interaction coupling parameter $b$. As an example taking $\Omega_{k}=0.01$ favored by observation, $\Omega_{D}=0.72$ for the present time, $\varepsilon=0.002$ and $b>0.32$ lead to $w_{D}<-1.01$. It is worth noting that the existence of the spatial curvature in this section does not lead to a considerable difference between the acceleration rate or the deceleration rate of the universe with respect to the flat case. However, it should be emphasize that the existence of a non-zero curvature in the universe has very important consequences on global topology of the universe and much subtle issues.

\section{DISCUSSION}

Recently, the vacuum energy of the Veneziano ghost field in a time-dependent background was proposed as a kind of $\mathrm{DE}$ candidate to explain the acceleration of the cosmic expansion. In this model, the energy density of the dark energy is proportional to the Hubble parameter $H$. In this paper we investigated GDE model in the BD framework. In this framework we studied the GDE in three distinct cases. At first, we generalized the GDE to BD theory in the absence of interaction between DM and GDE in a flat FRW background. In this case the EoS parameter was obtained and it was found that for $\Omega_{D}=0.72, \omega=10000$ and $\varepsilon=0.002$ the universe enters a phase of accelerated expansion at the late time. Another interesting result from this part is that one can generate a phantom-like EoS from a noninteracting GDE in a flat background, which is in contrast to the GDE in standard cosmology. For example, taking $\Omega_{D}=0.72, \omega=10000$ and $\varepsilon=0.007$ leads $w_{D}=-1.04$. In the next two sections we presented the interacting GDE in both flat and non-flat BD cosmology. In both of these sections we found that the universe can enter a phase of acceleration as well as it can cross the phantom line with suitable choice of constants. For example for $\Omega_{k}=0.01$ favored by observation, $\Omega_{D}=0.72$ for the present time, $\varepsilon=0.002$ and $b>0.35$ lead to $w_{D}<-1.05$. For all cases we plotted $w_{D}$ versus $\varepsilon$ and found that crossing from the phantom line is possible provided we take $\varepsilon<0.01$ and in the region with $\varepsilon>0.01$ we sometimes even cannot have a phase of acceleration. The important point is that such a constraint exist from the observational point of view [43] and we obtained such a condition theoretically. Also we investigated the deceleration parameter in different cases. For flat universe in the absence of interaction between DE and DM we found that with $\Omega_{D}=0.72$ for present time and choosing $\varepsilon=0.002$ and $\omega=10000$, we reach $q=-0.36$ which shows a consistent value in comparison with observation. Also this parameter have a consistent behavior in all cases of interest.

\section{Acknowledgments}

This work has been supported by Research Institute for Astronomy and Astrophysics of Maragha, Iran.

[1] A.G. Riess, et al., Astron. J. 116 (1998) 1009;

S. Perlmutter, et al., Astrophys. J. 517 (1999) 565;

S. Perlmutter, et al., Astrophys. J. 598 (2003) 102;

P. de Bernardis, et al., Nature 404 (2000) 955.

[2] D.N. Spergel et al., Astrophys. J. Suppl. 148, 175 (2003). 
[3] D.N. Spergel et al., astro-ph/0603449 (2006)

[4] M. Tegmark et al., Phys. Rev. D 69, 103501 (2004).

[5] M. Tegmark et al., Astrophys. J. 606, 702 (2004).

[6] C. Wetterich, Nucl. Phys B. 302, 668 (1988).

[7] B. Ratra and J. Peebles, Phys. Rev D 37, 321 (1988).

[8] T. Chiba, T. Okabe and M. Yamaguchi, Phys. Rev. D 62, 023511 (2000).

[9] C. Armendariz-Picon, V. Mukhanov, and P. J. Steinhardt, Phys. Rev. Lett. 85, 4438 (2000).

[10] C. Armendariz-Picon, V. Mukhanov, and P. J. Steinhardt, Phys. Rev. D 63, 103510 (2001).

[11] R.R. Caldwell, M. Kamionkowski, N.N. Weinberg, Phys. Rev. Lett. 91, 071301 (2003).

[12] B. Feng et al., Phys. Lett. B 607, 35 (2005).

[13] T. Padmanabhan, Phys. Rev. D 66, 021301 (2002).

J. S. Bagla, H. K. Jassal and T. Padmanabhan, Phys. Rev. D 67, 063504 (2003).

L. R. W. Abramo and F. Finelli, Phys. Lett. B 575165 (2003).

J. M. Aguirregabiria and R. Lazkoz, Phys. Rev. D 69, 123502 (2004).

Z. K. Guo and Y. Z. Zhang, JCAP 0408, 010 (2004).

E. J. Copeland, M. R. Garousi, M. Sami and S. Tsujikawa, Phys. Rev. D 71, 043003 (2005).

[14] M. Li, Phys. Lett. B 603, 1 (2004);

D. Pavon, W. Zimdahl, Phys. Lett. B 628 (2005) 206;

B. Wang, Y. Gong and E. Abdalla, Phys. Lett. B 624 (2005) 141;

A. Sheykhi, Class. Quantum Grav. 27 (2010) 025007.

[15] R. G. Cai, Phys. Lett. B 657 (2007) 228.

H. Wei and R. G. Cai, Phys. Lett. B 660 (2008) 113;

A. Sheykhi, Phys. Lett. B 680 (2009) 113;

A. Sheykhi, Phys. Lett. B 682 (2010) 329;

A. Sheykhi, Phys. Rev. D 81 (2010) 023525.

[16] P. Jordan, Schwerkraft und Weltall, Vieweg (Braunschweig) 1955.

[17] C. H. Brans , R. H. Dicke, Phys. Rev. 124, 925 (1961).

[18] B. Bertotti, L. Iess, and P. Tortora, Nature (London) 425 (2003) 374.

[19] C. Mathiazhagan and V.B. Johri, Class. Quantum Grav. 1 (1984) L29;

D. La and P.J. Steinhardt, Phys. Rev. Lett. 62 (1989) 376;

S. Das and N. Banerjee, arXiv:0803.3936.

[20] F. R. Urban and A. R. Zhitnitsky, Phys. Lett. B 688 (2010) 9 ;

Phys. Rev. D 80 (2009) 063001; JCAP 0909 (2009) 018;

Nucl. Phys. B 835 (2010) 135.

[21] N. Ohta, Phys. Lett. B 695 (2011) 41, arXiv:1010.1339.

[22] E. Witten, Nucl. Phys. B 156 (1979) 269.

G. Veneziano, Nucl. Phys. B 159 (1979) 213.

[23] C. Rosenzweig, J. Schechter and C. G. Trahern, Phys. Rev. D 21 (1980) 3388.

[24] P. Nath and R. L. Arnowitt, Phys. Rev. D 23 (1981) 473.

[25] K. Kawarabayashi and N. Ohta, Nucl. Phys. B 175 (1980) 477;

N. Ohta, Prog. Theor. Phys. 66 (1981) 1408.

[26] R.G. Cai, Z.L. Tuo, H.B. Zhang, arXiv:1011.3212

[27] A. Sheykhi, M.Sadegh Movahed, arXiv:1104.4713.

[28] G. Dvali, G. Gabadadze, M. Porrati, Phys. Lett. B 485, 208 (2000); G. Dvali, G. Gabadadze, Phys.Rev. D 63065007 (2001).

[29] M. Carena, J. Lykken, M. Park, J. Santiago, Phys. Rev. D 75 (2007) 026009; Masato Minamitsuji, Phys. Lett. B 684 (2010) 92.

[30] C. Deffayet, Phys. Lett. B 502 (2001) 199.

[31] C. L. Bennett, et al., Astrophys. J. Suppl. 148 (2003) 1;

D. N. Spergel, Astrophys. J. Suppl. 148 (2003) 175;

M. Tegmark, et al., Phys. Rev. D 69 (2004) 103501;

U. Seljak, A. Slosar, P. McDonald, JCAP 0610 (2006) 014;

D. N. Spergel, et al., Astrophys. J. Suppl. 170 (2007) 377.

[32] J. L. Sievers, et al., Astrophys. J. 591 (2003) 599;

C.B. Netterfield, et al., Astrophys. J. 571 (2002) 604;

A. Benoit, et al., Astron. Astrophys. 399 (2003) L25;

A. Benoit, et al., Astron. Astrophys. 399 (2003) L19.

[33] R. R. Caldwell, M. Kamionkowski, astro-ph/0403003.

B. Wang, Y. G. Gong, R. K. Su, Phys. Lett. B 605 (2005) 9.

[34] J. P. Uzan, U. Kirchner, G.F.R. Ellis, Mon. Not. R. Astron. Soc. 344 (2003) L65;

A. Linde, JCAP 0305 (2003) 002;

M. Tegmark, A. de Oliveira-Costa, A. Hamilton, Phys. Rev. D 68 (2003) 123523;

G. Efstathiou, Mon. Not. R. Astron. Soc. 343 (2003) L95;

J. P. Luminet, J. Weeks, A. Riazuelo, R. Lehou, J. Uzan, Nature 425 (2003) 593; 
G. F. R. Ellis, R. Maartens, Class. Quantum Grav. 21 (2004) 223.

[35] C.J. Feng, X. Z. Li and X. Y. Shen, arXiv:1105.3253

[36] A. Sheykhi, M.Sadegh Movahed, E. Ebrahimi, arXiv:1105.0325.

[37] A. Sheykhi, A. Bagheri, arXiv:1104.5271.

[38] M. Arik, M.C. Calik, Mod. Phys. Lett. A 21 (2006) 1241;

M. Arik, M.C. Calik, M.B. Sheftel, gr-qc/0604082

[39] C. L. Bennett, et al., Astrophys. J. Suppl. 148 (2003) 1;

D. N. Spergel, Astrophys. J. Suppl. 148 (2003) 175;

M. Tegmark, et al., Phys. Rev. D 69 (2004) 103501;

U. Seljak, A. Slosar, P. McDonald, JCAP 0610 (2006) 014;

D. N. Spergel, et al., Astrophys. J. Suppl. 170 (2007) 377.

[40] N. Banerjee, D. Pavon, Phys. Lett. B 647 (2007) 447;

A. Sheykhi, Phys. Lett. B 681 (2009) 205.

[41] C.M. Will, Theory and Experiment in Gravitational Physics, Cambridge University Press, Cambridge, 1993.

[42] V. Acquaviva, L. Verde, JCAP 0712 (2007) 001.

[43] F. Wu and X. Chen, arXiv:0903.0385

[44] R. A. Daly et al., Astrophysics J. 677 (2008) 1.

[45] Bertolami O, Gil Pedro F and Le Delliou M 2007 Phys. Lett. B 654165.

[46] S. Tsujikawa, M. Sami, Phys. Lett. B 603 (2004) 113.

[47] Q. G. Huang and M. Li, J. Cosmol. Astropart. Phys. JCAP08(2004)013.

[48] T. P. Waterhouse and J. P. Zipin arXiv:0804.1771. 